Proceedings of the International School and Conference on Optics and Optical Materials, ISCOM07, Belgrade, Serbia, September 3-7, 2007

\title{
Silicon Photonic Waveguides for Near- and Mid-Infrared Regions
}

\author{
S. Stankovic ${ }^{a, *}$, M. Milosevic ${ }^{a}$, B. Timotijevic ${ }^{b}$,

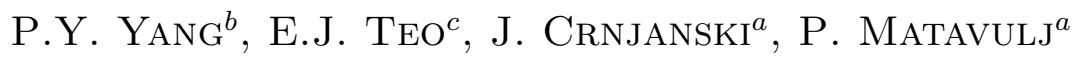 \\ AND G.Z. MASHANOVICH ${ }^{b}$ \\ ${ }^{a}$ Faculty of Electrical Engineering, University of Belgrade, Serbia \\ ${ }^{b}$ Advanced Technology Institute, University of Surrey, Guildford, UK \\ ${ }^{c}$ Department of Materials Science and Engineering \\ National University of Singapore, Singapore
}

The basic building block of every photonic circuit is a waveguide. In this paper we investigate the most popular silicon waveguide structures in the form of a silicon-on-insulator rib waveguide. We also analyse two structures that can find applications in mid- and long-wave infrared regions: free-standing and hollow core omnidirectional waveguides.

PACS numbers: 42.82.--m, 42.82.Et

\section{Introduction}

Two most common basic channel waveguide structures in silicon photonics, a field that is experiencing a dramatic increase in interest due to emerging application areas and several high profile successes in device and technology development, are rib and strip silicon-on-insulator (SOI) waveguides. Rib waveguides give more flexibility regarding dimensions for single mode and zero-birefringence operation. They can also have larger cross-sections facilitating coupling from optical fibres. These waveguides have large bend radii, however, making photonics circuits larger, less efficient and more expensive. The design rules for small rib waveguides $(1.0<H<1.5 \mu \mathrm{m})$ have been reported, but only with air top cladding [1]. The influence of the oxide top cover has been analysed for larger rib waveguides with height of $H=2.2 \mu \mathrm{m}$ [2]. In this paper, the influence of the top oxide cover in a SOI rib waveguide with $H=1.3 \mu \mathrm{m}$ has been analysed as polarisation independent ring resonators and multi-stage resonators based on such waveguides have been reported [3, 4].

*corresponding author; e-mail: stevans@etf.bg.ac.yu 
SOI waveguides are not suitable for longer wavelengths (except in the 3-3.5 $\mu \mathrm{m}$ range) due to the absorption of the silicon dioxide insulating layer [5]. Mid- and long-wave infrared (LWIR) spectra are of interest in a variety of applications, such as sensing, imaging or medical applications. In this paper we present an improved free-standing waveguide which has air cladding and offers potentially low-loss propagation in the $1.2-6 \mu \mathrm{m}$ and $24-100 \mu \mathrm{m}$ wavelength regions. In conventional waveguides, the core refractive index is higher than the cladding refractive index. Due to this fact, their sensor applications are somewhat limited. However, hollow channel waveguides whose walls consist of multilayer coatings can be very useful for sensor applications as their core can be infilled with gases or liquids. Other potential applications of these waveguides include tight turning radii, high power transmission, and dispersion compensation. In this paper, we analyse one such structure that can be used in the $3-3.5 \mu \mathrm{m}$ range.

\section{Rib waveguides}

The modal birefringence is defined as the difference between the effective indices of the two orthogonally polarised modes, quasi-TE and quasi-TM: $\Delta N_{\text {eff }}=$ $N_{\text {eff }}^{\mathrm{TE}}-N_{\mathrm{eff}}^{\mathrm{TM}}$. To achieve zero-birefringence, an optimisation of rib waveguide dimensions is necessary [1]. The total birefringence is, however, the sum of the geometrical birefringence and stress induced birefringence [2]. The rib waveguide analysed in this section has a buried oxide (BOX) layer of $1 \mu \mathrm{m}$ in thickness, rib width of $1.099 \mu \mathrm{m}$, height of $1.304 \mu \mathrm{m}$, and etch depth of $0.769 \mu \mathrm{m}$ (Fig. 1a). The stress in the upper $\mathrm{SiO}_{2}$ layer produces a stress distribution within and near the $\mathrm{Si}$ rib, which in turn causes a change of the refractive index in both materials due to the photoelastic effect. We assume that the thickness of the upper cladding film on the rib sidewalls is $70 \%$ of that on the top (Fig. 1a). There is additional oxide layer, that of the hard mask used during the etching of the rib. Its thickness is $0.274 \mu \mathrm{m}$. Sidewall angle is assumed to be 8 degrees.

The stress induced birefringence can be expressed as [6]:

$$
\begin{aligned}
& \Delta n_{x}=n_{x}-n_{0}=-C_{1} \sigma_{x}-C_{2}\left(\sigma_{y}+\sigma_{z}\right), \\
& \Delta n_{y}=n_{y}-n_{0}=-C_{1} \sigma_{y}-C_{2}\left(\sigma_{x}+\sigma_{z}\right),
\end{aligned}
$$

where $\sigma_{x}, \sigma_{y}$, and $\sigma_{z}$ are principal components of the relative stress tensor, $n_{x}$ and $n_{y}$ are components of the material's refractive index, $n_{0}$ is the refractive index without stress, and $C_{1}$ and $C_{2}$ are the stress-optic constants related to the Young modulus $(E)$, Poisson's ratio $(\nu)$, and the photoelastic tensor elements $\left(p_{11}\right.$ and $\left.p_{12}\right)$ as: $C_{1}=\left(n^{3} / 2 E\right)\left(p_{11}-2 \nu p_{12}\right)$ and $C_{2}=\left(n^{3} / 2 E\right)\left[-\nu p_{11}+(1-\nu) p_{12}\right]$

Stress distribution in Si rib waveguide is calculated by the finite element method, which is implemented in COMSOL software package [7]. The refractive indices, at the wavelength of $1550 \mathrm{~nm}$, for the silicon guiding layer and BOX used in the model are 3.476 and 1.444 , respectively. The thermal expansion coefficients for $\mathrm{Si}$ and $\mathrm{SiO}_{2}$ layers are $3.6 \times 10^{-6} \mathrm{~K}^{-1}$ and $5.4 \times 10^{-7} \mathrm{~K}^{-1}$, whilst the difference 


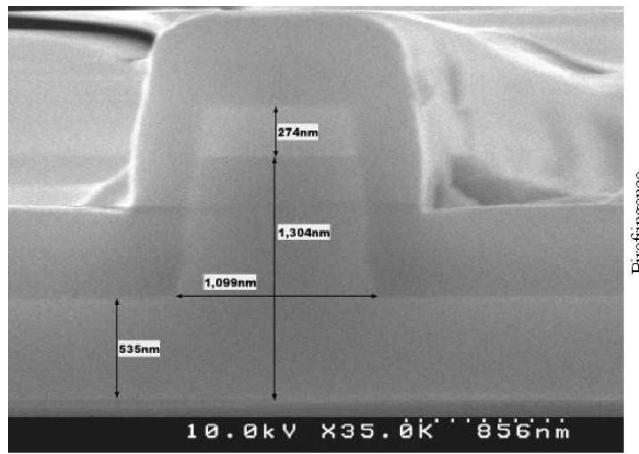

(a)

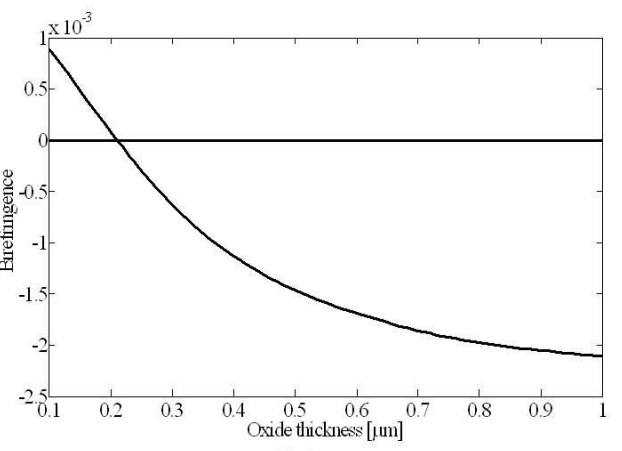

(b)

Fig. 1. (a) Scanning electron microscopy (SEM) of SOI rib waveguide cross-section, (b) waveguide birefringence $\Delta N_{\text {eff }}$ as a function of the upper cladding oxide thickness.

between the operating and reference temperature is assumed to be $-980 \mathrm{~K}$. Photoelastic tensor elements, Poisson's ratios, and Young's modulus for $\mathrm{Si}$ and $\mathrm{SiO}_{2}$ are identical to those used in [2].

Figure $1 \mathrm{~b}$ shows the influence of the oxide cover on the birefringence. For air cover, the total birefringence is equal to the geometrical birefringence and it is positive. The oxide cover reduces the total birefringence and for thick oxides it becomes negative. It can be seen that for the oxide thickness of $\approx$ $220 \mathrm{~nm}$ zero-birefringence can be achieved. Therefore, by stress engineering, zero-birefringence can be achieved for a waveguide that has otherwise relatively large birefringence.

\section{Hollow-core and free standing waveguides}

Hollow-core waveguides feature an air-filled core surrounded by walls made of multilayer coatings, designed to confine the propagating optical signal within the core (Fig. 2). Since the light propagates through air, there are virtually no dispersion and problems with temperature dependence of the refractive index. The hollow-core waveguides can be also very useful for sensing applications as their core can be filled with gases or liquids. MIR wavelength region is particularly interesting for gas sensing as many gases exhibit bands of absorption lines in this part of the spectrum. In this section we analyse hollow-core omnidirectional waveguides (HOWs) with the Bragg-mirror cladding (Fig. 2), optimised for mid-infrared (MIR) spectrum. Due to a large difference in the refractive indices of $\mathrm{Si}$ and $\mathrm{SiO}_{2}$, small propagation losses can be achieved with the waveguides having no more than $5 \mathrm{Si} / \mathrm{SiO}_{2}$ bilayers in the cladding. We have used a $3 \mathrm{D}$ beam propagation method [8] to calculate waveguide propagation losses for several different values of the core dimensions and overall number of bilayers in the cladding. All simulations were carried out at the wavelength of $\lambda=3.39 \mu \mathrm{m}$. For the sake of better comparison, core dimensions (width and height) were expressed as multiples of the wavelength. 


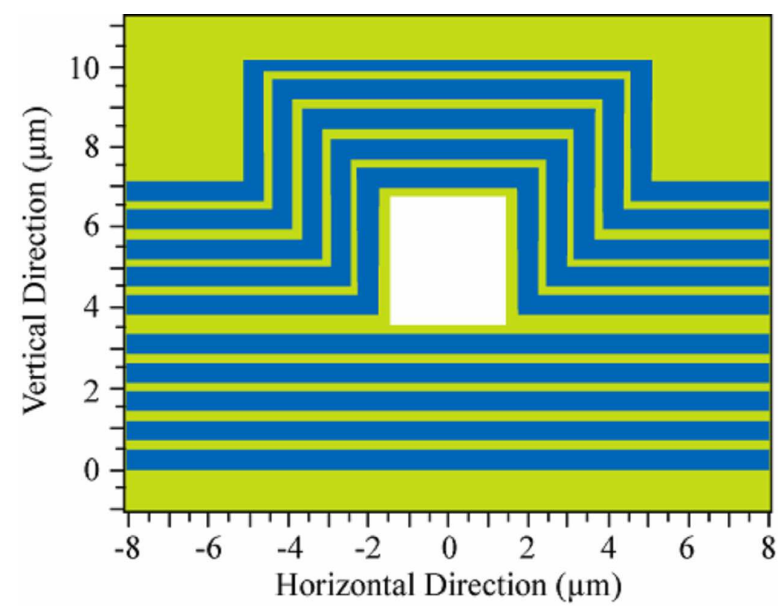

Fig. 2. Hollow-core waveguide with square cross-section.

TABLE

Propagation losses calculated for hollow-core waveguides with claddings based on $\mathrm{Si} / \mathrm{SiO}_{2}$ bilayers, at the wavelength of $\lambda=3.39 \mu \mathrm{m}$.

\begin{tabular}{c|c|c}
\hline \hline $\begin{array}{c}\text { Core dimensions } \\
\text { [in wavelengths] }\end{array}$ & $\begin{array}{c}\text { Number of } \\
\text { bilayers }\end{array}$ & $\begin{array}{c}\text { Propagation loss } \\
{[\mathrm{dB} / \mathrm{cm}]}\end{array}$ \\
\hline $2 \lambda \times 2 \lambda$ & 4 & 4.78 \\
$2 \lambda \times 2 \lambda$ & 5 & 2.41 \\
$3 \lambda \times 3 \lambda$ & 4 & 0.66 \\
$3 \lambda \times 3 \lambda$ & 5 & 0.17 \\
$4 \lambda \times 4 \lambda$ & 4 & 0.06
\end{tabular}

Calculated propagation losses for several waveguide structures are presented in Table.

These results show that waveguides with only 4 or 5 bilayers in the cladding, and with relatively large core dimensions of no less than three wavelengths in both width and height, could exhibit propagation losses below $1 \mathrm{~dB} / \mathrm{cm}$. It can be seen from Table that the core dimension has greater impact on propagation losses than the overall number of bilayers in the cladding. Even for waveguides with only 4 bilayers in the cladding, the propagation loss is reduced almost 10 times by increasing the core size from $3 \lambda \times 3 \lambda$ to $4 \lambda \times 4 \lambda$ cross-section area.

A free standing silicon waveguide has an air cladding (Fig. 3), and therefore it is a viable structure for long wavelength applications. We have fabricated the waveguides by using the proton beam writing (PBW) technique. In order to fabricate free standing waveguides, two different ion energies are required. A high energy implant is used to create pillars upon which the waveguides will be supported. A lower energy implant is subsequently used to create the waveguides [9]. 
There are several potential advantages of PBW. It is a direct write process which eliminates the need for a potentially costly mask. Furthermore, prototype devices which require small structural modifications can be produced by simply modifying the scan of the microbeam whereas modifications to a device using photolithographic methods would require the layout and fabrication of an additional mask. Also, the protons are deposited in the material in a well-defined range thereby allowing for good vertical control of the fabrication of devices. Furthermore, the high energy protons deviate little from the ideal straight path due to multiple small-angle Coulomb scattering. This can allow fabrication of high aspect ratio structures.

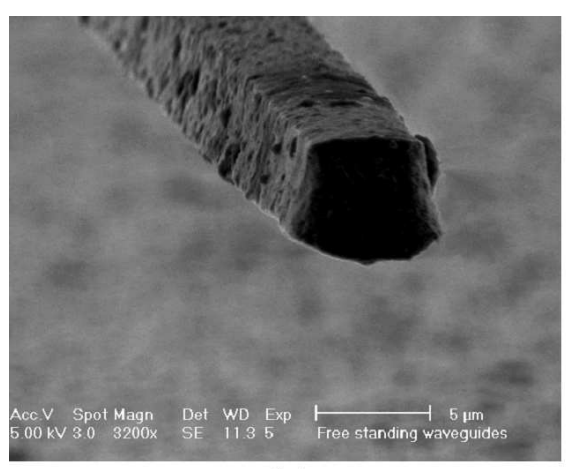

(a)

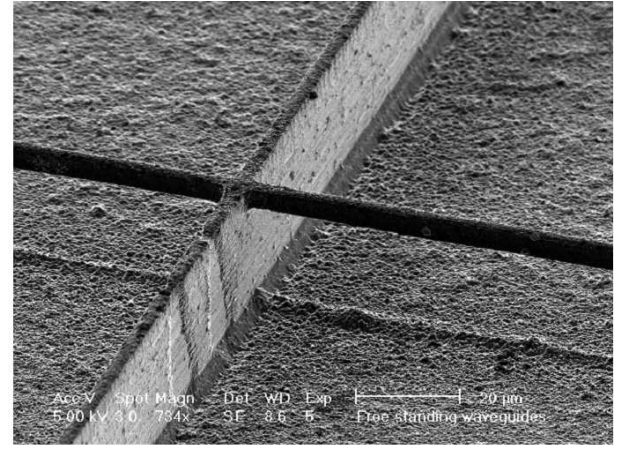

(b)

Fig. 3. (a) Cross-section of $0.5 \mathrm{MeV}$ protons free-standing waveguide; (b) free-standing waveguide and supporting pillar.

We have already fabricated free standing waveguides which had a "teardrop" shape with a height of $17 \mu \mathrm{m}$, and width of $3.7 \mu \mathrm{m}$ at the top and $8 \mu \mathrm{m}$ at the bottom [9]. In this work, freestanding waveguide structures with improved cross-section are fabricated by employing $0.5 \mathrm{MeV}$ proton energy. A well focused proton beam of $200 \mathrm{~nm}$ resolution is used to irradiate the Si sample. The beam is magnetically scanned in the $x$-direction, which determines the width of the waveguide, in this case $5 \mu \mathrm{m}$, whilst simultaneously applying a constant linear perpendicular motion in the $y$-direction, provided by an Exfo Burleigh Inchworm stage. The proton fluence delivered to the sample is $5 \times 10^{14}$ protons $/ \mathrm{cm}^{2}$. After irradiation, the sample is electrochemically etched in a 1:1 electrolyte mixture of $48 \% \mathrm{HF}$ and ethanol at a current density of $40 \mathrm{~mA} / \mathrm{cm}^{2}$. Once the silicon becomes porous, it can be removed by a dilute potassium hydroxide solution, producing a waveguide with an approximately square profile in the cross-section. A SEM image of the waveguide cross-section is shown in Fig. 3a. The waveguide has a height of $5.5 \mu \mathrm{m}$ and width of $5 \mu \mathrm{m}$ at the top and $6 \mu \mathrm{m}$ at the bottom. It can be seen from Fig. 3 that the waveguide surface is rough, and that is probably the main reason for high propagation losses of $\approx 10 \mathrm{~dB} / \mathrm{cm}$. Annealing of the waveguide can reduce the surface roughness and lattice damage caused by the proton irradiation. 


\section{Conclusions}

We have presented design guidelines for conventional SOI rib waveguides which are the most common structures for near infrared silicon photonic applications. The influence of the top oxide cover on the zero-birefringence operational regime is analysed for a rib waveguide with height of $1.3 \mu \mathrm{m}$. It has been shown that for top oxide cladding thickness of $\approx 200 \mathrm{~nm}$ zero-birefringence can be achieved for a waveguide with otherwise large birefringence. The core size of the hollow-core omnidirectional waveguide is very important for reducing the propagation losses. For larger dimensions of the core, propagation losses can be significantly reduced, and also the number of bilayers in the Bragg cladding. This conclusion is in agreement with the analysis of ARROW hollow-core waveguides. Finally, a free-standing silicon waveguide, which is a candidate for MIR and LWIR applications, with improved cross-sectional dimensions of $\approx 5.5 \times 5.5 \mu \mathrm{m}$ has been fabricated.

\section{References}

[1] S.P. Chan, C.E. Png, S.T. Lim, G.T. Reed, V.M.N. Passaro, J. Lightwave Technol. 23, 2103 (2005).

[2] W.N. Ye, D.-X. Xu, S. Janz, P. Cheben, M.-J. Picard, B. Lamontagne, N.G. Tarr, J. Lightwave Technol. 23, 1308 (2005).

[3] W.R. Headley, G.T. Reed, S. Howe, A. Liu, M. Paniccia, Appl. Phys. Lett. 85, 5523 (2004).

[4] B.D. Timotijevic, F.Y. Gardes, W.R. Headley, G.T. Reed, M.J. Paniccia, O. Kohen, D. Hak, G.Z. Masanovic, J. Opt. A, Pure Appl. Opt. 8, S473 (2006).

[5] R.A. Soref, S.J. Emelett, W.R. Buchwald, J. Opt. A, Pure Appl. Opt. 8, 840 (2006).

[6] M. Huang, Int. J. Sol. Structures 40, 1615 (2003).

[7] www.comsol.com.

[8] www.rsoftdesign.com.

[9] P.Y. Yang, G.Z. Mashanovich, I. Gomez-Morilla, W.R. Headley, G.T. Reed, E.J. Teo, D.J. Blackwood, M.B.H. Breese, A.A. Bettiol, Appl. Phys. Lett. 90, 241109 (2007). 